\title{
Scanning Electron Microscopic Study of Cholesterol Gallstones
}

\author{
Takuro Ogata and Fumio Murata \\ Department of Surgery, Okayama University Medical School, \\ Okayama
}

Ogata, T. and Morata, F. Scanning Electron Microscopic Study of Cholesterol Gallstones. Tohoku J. exp. Med., 1971, 104 (1), 25-44 - The latest techniques in scanning electron microscopy have been applied to this study. In cholesterol gallstones, the fracture surface generally shows a crystalline plane radiating pattern, often showing dendritic growth, around the nucleus. On the surface or fracture plane of a cholesterol stone, parallelogram crystals or corrugated crystals are sometimes seen. In higher magnification, the surface of a crystalline plane is seen to be composed of lamellae of about $200 \AA$ in thickness, which is frequently associated with spiral growth. In view of the fact that a number of linear boundaries are just visible near the center of each spherulite, it appears that, in the multinuclear stones, cyrstallization starts at various points or nuclei and spreads out from these points. These results support the view that cholesterol crystals proceed from the center toward the periphery. - scanning electron microscopy; cholesterol gallstone

The cause of gallstone formation has not yet been clarified, and studies on the structure of gallstones have been thought to be important to clarify their etiology and growth. Information on this subject up to the present has been derived from observations by light (Nomamoto 1944, Sakai 1960), polarization (Matsunaga 1966) and mineralogical microscopes (Endo 1962). However, these studies failed to clarify the fine structure of gallstones because it was beyond the resolving power of these microscopes. On the other hand, the scanning electron microscope, with its huge power of magnification and great depth of focus, can demonstrate the fine structure of the stones.

In the present study using a scanning electron microscope, very precise observations have been made on the surface and fracture plane of cholesterol stones. These observations provide a satisfactory explanation of the etiology and growth of cholesterol gallstones.

\section{Materials and Methods}

Forty cholesterol stones, obtained from 30 patients with cholelithiasis in our surgical clinic, were used in this study. After removal of the stones, they were washed with distilled water, then most of them were fixed in $10 \%$ formalin for 3 hours. Some of them were unfixed and used for observation at higher magnification. The stones were dried in air for at least one week. For observation of fracture planes, the stones were split with the tip of

Received for publication, October 17, 1970. 
a screw-driver. The external surface and fracture plane of the stones were usually coated with carbon and gold in a vacuum at a thickness of about $200 \mathrm{~A}$ each, but for the observation at higher magnifications the thickness of coating was about $100 \AA$ each. The specimens were observed and photographed with a JSM-2 type scanning electron microscope (Japan Electron Optics Laboratory Co., Ltd.) using a beam accelerating voltage of 16 or $25 \mathrm{KV}$. For the determination of the chemical composition of stones, infrared spectroscopy was applied.

\section{RESULTS}

\section{a) General structure:}

Cholesterol gallstones have some common features. To the naked eye, the fracture plane shows crystalline planes in a radiating structure, often arranged in a concentric zonal pattern. It has various hues, but usually the radial parts are yellowish-white, the zonal parts yellowish-black, and the central part brown.

In the scanning electron micrographs, the fracture plane has basically a sheet structure of plate-like layers, in which one layer is tightly joined to the layer below and the other layers are more or less arranged in radiating form, though the growth of crystals is rather irregular and the tendency toward dendritic growth increases toward the serrated edge (Fig. 1).

Another conspicuous feature is a phenomenon similar to twinning, that is, some parts of the crystal which developed straight from the center change their direction at an angle of about $25^{\circ}$, while the other parts keep the original direction (Fig. 2).

When the cut surfaces which appear with the naked eye to have a cyclical pattern are observed more closely, the shape of the crystal is seen to be essentially similar to those with a radial pattern, whereas those with a plate-like crystalline arrangements are different from them. Figs. 4 and 5 show boundaries in which, on either side of a boundary, the growth of crystals in the two planes A and B are at different angles. The boundary separating the two areas may be regarded as only a line formed by the edge dislocation. In Fig. 4 the dislocation line is nearly perpendicular to the direction of growth in the central part, and in Fig. 5 it is slightly deviated.

The innermost part or so-called "nucleus" shows to the naked eye an irregular splitting pattern with a dark brown color in the stone. In the scanning electron micrograph, the nuclear portion is filled with very small foliate or layered cholesterine crystals that are mixed up together and loosely joined (Figs. 1 and 3). Together with these, bilirubine granules and undetermined substances are also aggregated.

The external surface of the stones appears gently nodular or bulging in shape to the naked eye. Under the scanning electron microscope, the fine structure of the crystals on the outermost external surface has various shapes, but generally it consists of parallel columnar projections arranged like cogs on a cogwheel with multiple cutting edges or points distributed around its circumference (Figs. 6 and 8). Furthermore, these projections are composed of a multiplicity of fine-layered 
lamellae of $200 \AA ̊$ thickness (Fig. 9).

In some stones, plate-like crystals shaped in parallelogram-blocks, which consist of finely multilayered crystal sheets, are arranged over the surface (Fig. 7). The obtuse angle of such a parallelogram is about $120^{\circ}$. It is interesting that smaller crystals develop from a large base one (Figs. 7 and 8). In this case, monolayered island develops only on the top of the crystal; this is likely to be an indication of an epitaxial alignment, where a new layer was about to be formed.

\section{b) Growth spiral:}

In a well-preserved sample, spiral growth can often be observed on the surface of a cholesterol crystal. As seen in Figs. 10 and 11, the ledge will curl and form a spiral. The exact shape of the spiral will depend upon the direction taken by the crystal during its process of growth. For the demonstration of growth spirals, it is indispensable for the sample to be unfixed and to have gold and carbon coatings of no more than $100 \AA$ each, because each layer of the spiral is only about $200 \AA$ in thickness; therefore the sample tends to be destroyed by the electron beam.

\section{c) Multinuclear stones:}

A multinuclear stone is an assemblage of a number of small cholesterol crystallines, each of which independently formed a radial cholesterol structure around a nucleus in its central portion (Fig. 12). Crystallizations started simultaneously at various points termed nuclei and proceeded rapidly towards outside from these points. The growth fronts emitted from two different sources do not interfere with each other like ordinary wave fronts. Where two different crystals meet, they fuse and annilate each other over the common portion on a straight line. Examples can be seen in Figs. 12 and 13. This phenomenon supports the view that cholesterol crystals proceed from the center toward the periphery like the spherulitic crystals of polyethylene (Fig. 14).

\section{Discussion}

This scanning electron microscopic study has defined the ultrastructure of cholesterol crystals in pure cholesterol gallstones, showing that they are primarily composed of plate-like crystals shaped in parallelogram or corrugated form and that their over-all appearance shows a radiating pattern from the center.

Previous studies by light, polarization, and mineralogical microscopes had divided the cholesterol crystalline structures of gallstones into radial and stratified patterns which were generally composed of plate-like, fan-like, needle-like or foliate crystals.

In cholesterol gallstones, plate-like crystals parallelogrammic in shape are often seen (Fig. 7). The obtuse angle of this parallelogram is about $120^{\circ}$ and nearly corresponds to the $\beta$ of cell constants* of cholesterin crystals. So it may be

\footnotetext{
* According to Wilson (1956), the unit cell dimensions of cholesterol crystal are $a=14.0 \mathrm{~A}$, $\mathrm{b}=10.46 \AA, \mathrm{c}=37.8 \AA, \alpha=94^{\circ}, \beta=117^{\circ} 30^{\prime}, \gamma=90^{\circ}$.
} 
considered that the upper surface of the crystals corresponds to the $(010)$ plane. Thus, it is reasonable to assume that parallelogram shaped crystals in cholesterol gall stones are formed under ideal conditions for crystallization.

In corrugated crystals (Fig. 8), however, crystallization has probably taken place under less ideal conditions. Surface corrugations appear to represent a terminal stage of crystallization as seen in many polymer crystals.

Even under the naked eye, concentric lamina and zonal bands can be observed in the typical cholesterol stone. In compound stones composed of cholesterol and bilirubin, structural differences exist among individual stones as reported in previous papers (Ogata and Murata 1970, a, b). However, in cholesterol stones the crystal structure is always the same, but the direction in which crystals are arranged differs from zone to zone (Figs. 4 and 5).

There is no universally accepted or authoritative theory on the formation and growth of cholesterol gallstones. The major point of disagreement among the several investigations concerns the process of growth of the cholesterol stone, particulary whether the crystallization takes place from the center to the periphery or vice versa.

Naunyn (1921) insisted that precipitation of cholesterol and protein occurs first and forms the so-called 'nucleus', then cholesterol crystals spread from the nucleus outward.

Sweet (1935) asserted, however, that the outer layer is formed first, then crystallization proceeds to the center as observed in ice crystal formation. From $\mathrm{X}$-ray diffraction study, Tamura (1943) found that crystals at the peripheral part of the stone are smaller in size than those in the central part. He suggested that smaller crystals are formed rapidly, while larger ones are formed slowly; thus the peripheral crystals form first, then crystallization proceeds towards the center.

From the present investigation, in view of the following facts, we may now confidently assert that the cholesterol gallstone develops from the center to the periphery.

1) Dendrification or branching of the crystals is always observed to take place from the center toward the periphery, but never in the opposite direction.

2) On the surface of a cholesterol stone where growth is proceeding, smaller crystals are observed emerging from large base crystals on the surface.

3) In a multinuclear stone, the appearance of spherical growth is similar to that of spherulitic crystals of polyethylene described by Geil (1963). A number of linear boundaries, at which the growth of crystals terminates, are observed. This fact also supports the view that the cholesterol stone is grown from the center outward.

The central portion of a cholesterol stone, which is called "nucleus", has a complicated structure; it contains very small cholesterol crystals of various shapes arranged at random, bilirubin granules, and other undetermined structures. It is difficult to determine the precise mechanism of the formation of this "nucleus" from 
the above findings. But the following assumption may be made: at first a lump of cholesterol, bilirubin and protein etc. is aggregated from the bile as suggested by Naunyn (1921); then the crystallization proceeds within these lumps. As mentioned above, the cholesterol crystals inside this central portion are undeveloped and remain very small, possibly because their growth has been interrupted by a stoppage in the supply of cholesterol from the bile, while crystals situated outside this portion continue their growth and form a spherulite because they are in direct contact with the bile and obtain cholesterol.

The mechanism of cholesterol precipitation in the cholesterol gallstone has not yet been completely clarified. Applying the dislocation theory of Taylor, Frank (1949) explained that spiral growth is seen in the polymer crystal when the crystal is formed very slow at low supersaturation. A circular spiral structure with $200 \AA$ thickness was demonstrated on the surface of a large number of cholesterol crystals. Thus, spiral growth is one of the modes of growth of cholesterol crystals. The general appearance of the spiral growth in a cholesterol stone is somewhat irregular as compared with that observed in polymer crystals. This may be attributable to the fact that crystallization occurs under complicated conditions in the bile.

\section{Acknowledgment}

We wish to acknowledge the advice of Prof. S. Tanaka and Prof. S. Haisa. We are also greatly indebted to Drs. A. Kubotsu and J. Ukita, T. Mochizuki and K. Tsuboi of Kurashiki Rayon Co. for their kind assistance in scanning electron microscopy, and to Dr. H. Zaitsu of Niigata University Medical School for infrared spectroscopy.

\section{References}

1) Endo, S. A mineralogical investigation of gall stone. Tohoku J. exp. Med. 1962, 76, 326-349.

2) Frank, F.C. The influence of dislocation on crystal growth. Discussions Faraday Society. 1949, 5, 48-54.

3) Geil, P.H. Polymer single crystals. Interscience Publishers New York. 1963, 224.

4) Matsunaga, M. Mechanism in the formation of gallstones. Nippon Geka Gakkai Zasshi (Jap.), 1966, 67, 1005-1048.

5) Naunyn, B. Die Gallenstein, ihre Entstehung und ihre Bau. Mitteil. Grenzgeb. Med. Chir., 1921, 33, 1-54.

6) Nomamoto, S. Microscopic studies of the structure of gallstones. Igaku Kenkyu (Jap.), 1944, 18, 415-438.

7) Ogata, T. \& Murata, F. (a) A scanning electron microscopic study on the surface and cut-surface structure of human gall-, pancreas-, and urinary stones. Jap. J. clin. Electron Microscopy., 1970, 2, p. 420.

8) Ogata, T. \& Murata, F. (b) Scanning electron microscopic studies of the ultrastructure of human bile, pancreas and urinary stones. Rinsho Geka (Jap.), 1970, 25, 761-770.

9) Sakai, H. Microscopic studies of gall-stones. Nippon Geka Gakkai Zasshi (Jap.), 1960, 60, 2091-2108.

10) Sweet, J.E. The formation of gallstones. Ann. Surg., 1935, 150, 624-632.

11) Tamura, K. Consideration for mechanism of formation of cholesterol stones. Nippon Geka Gakkai Zasshi (Jap.), 1943, 44, 42-46.

12) Wilson, A.J.C. Structure Reports. N.V.A. Oosthoek's Uitgevers MIJ Utrecht. 1956, 8,336 . 


\section{Explanation of Figures}

Figures 1 to 13 show scanning electron micrographs of cholesterol gallstones. Infrared spectroscopic examination shows that all these stones are pure or almost pure cholesterol stones.

Fig. 1. Lower magnification of the fracture surface of a radiate cholesterol stone obtained from the gallbladder of a 31-year-old female. An array of plate-like crystals can be seen radiating from the nucleus $(\mathrm{N})$ to the periphery. In the nucleus, an amorphous, muddy-like substance and small crystals are intermingled. $\times 125$. 


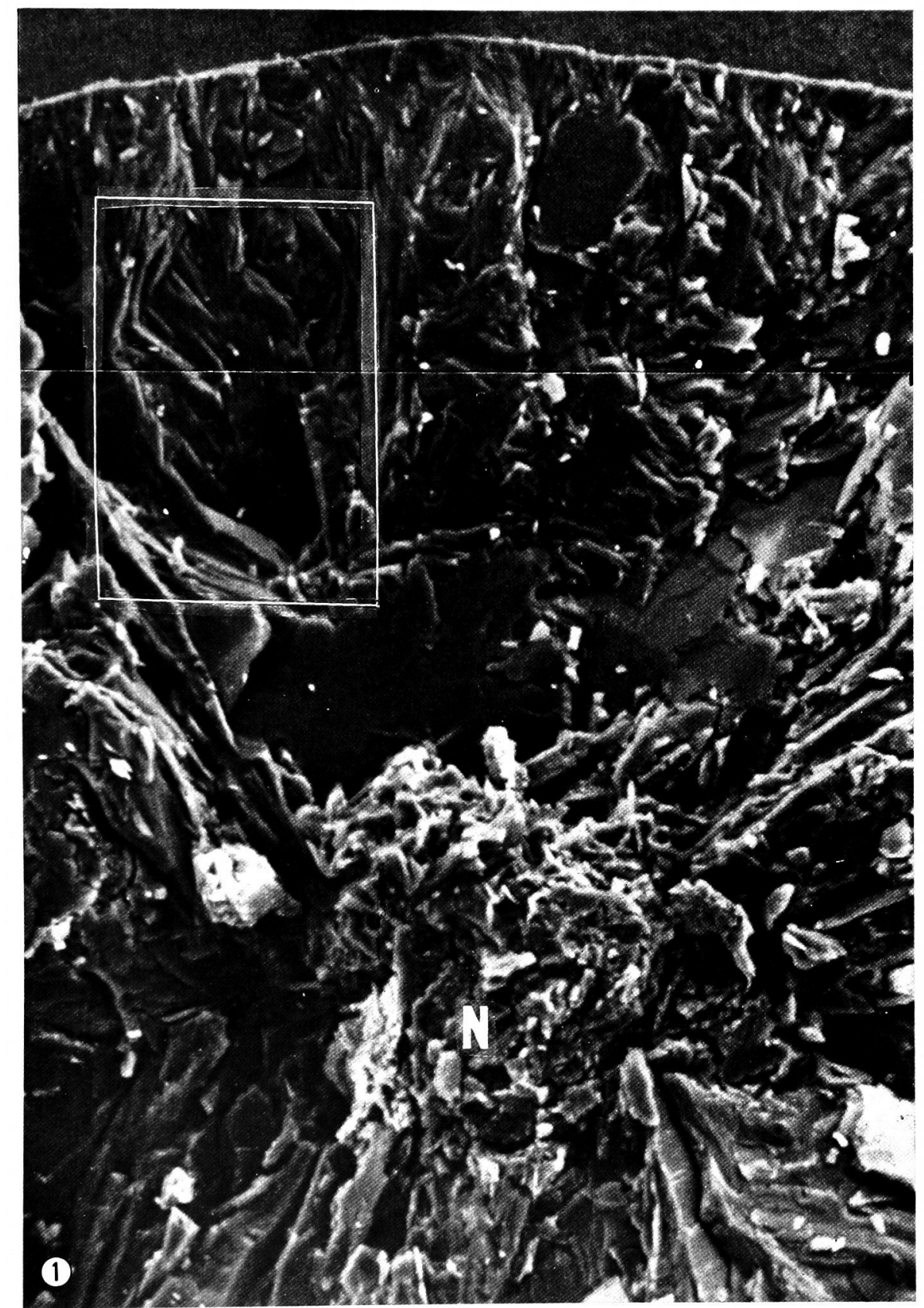


Fig. 2. Higher magnification of the boxed area of Fig. 1. Note that some parts (B) of crystal which developed straight out from the center (A) happened to bend at an angle of $25^{\circ}$, while others continued in the same straight direction (C). $\times 410$.

Fig. 3. Higher magnification of the central portion, the so-called nucleus of Fig. 1, illustrating an irregular structure which consists of loosely mixed small folliate or plate-like cholesterol crystals (C) and a muddy amorphous substance (S) possibly bilirubine. $\times 1,100$. 


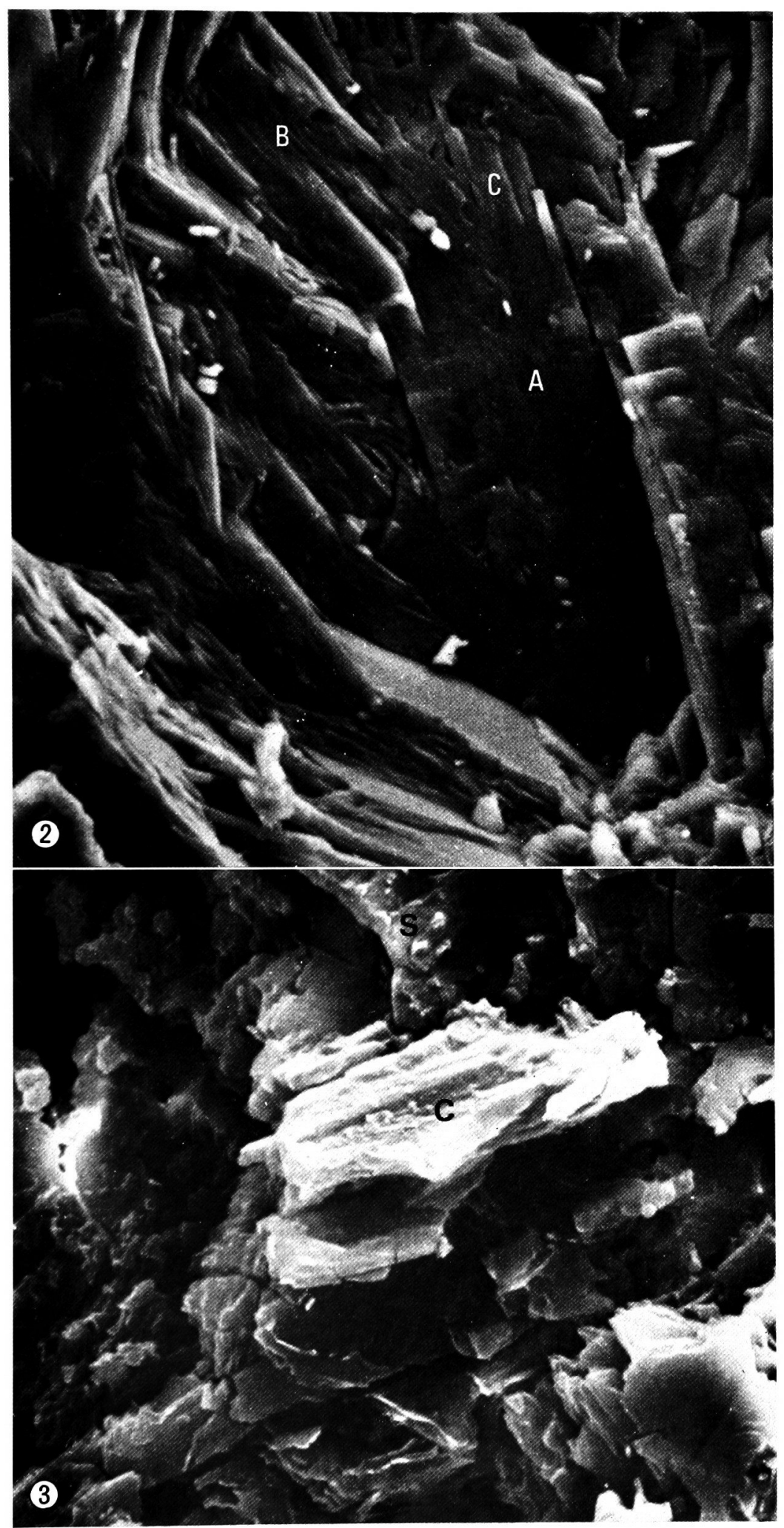


Figs. 4 and 5. Lower magnification of fracture plane of cholesterol stones. Portions which appear cyclically consist of plate-like crystallines. Note the discloation line between $A$ and B, showing more or less perpendicular distortion (Fig. 4) and/or a slight deviation (Fig. 5). Fig. 4: $\times 70$. Fig. 5: $\times 45$. 


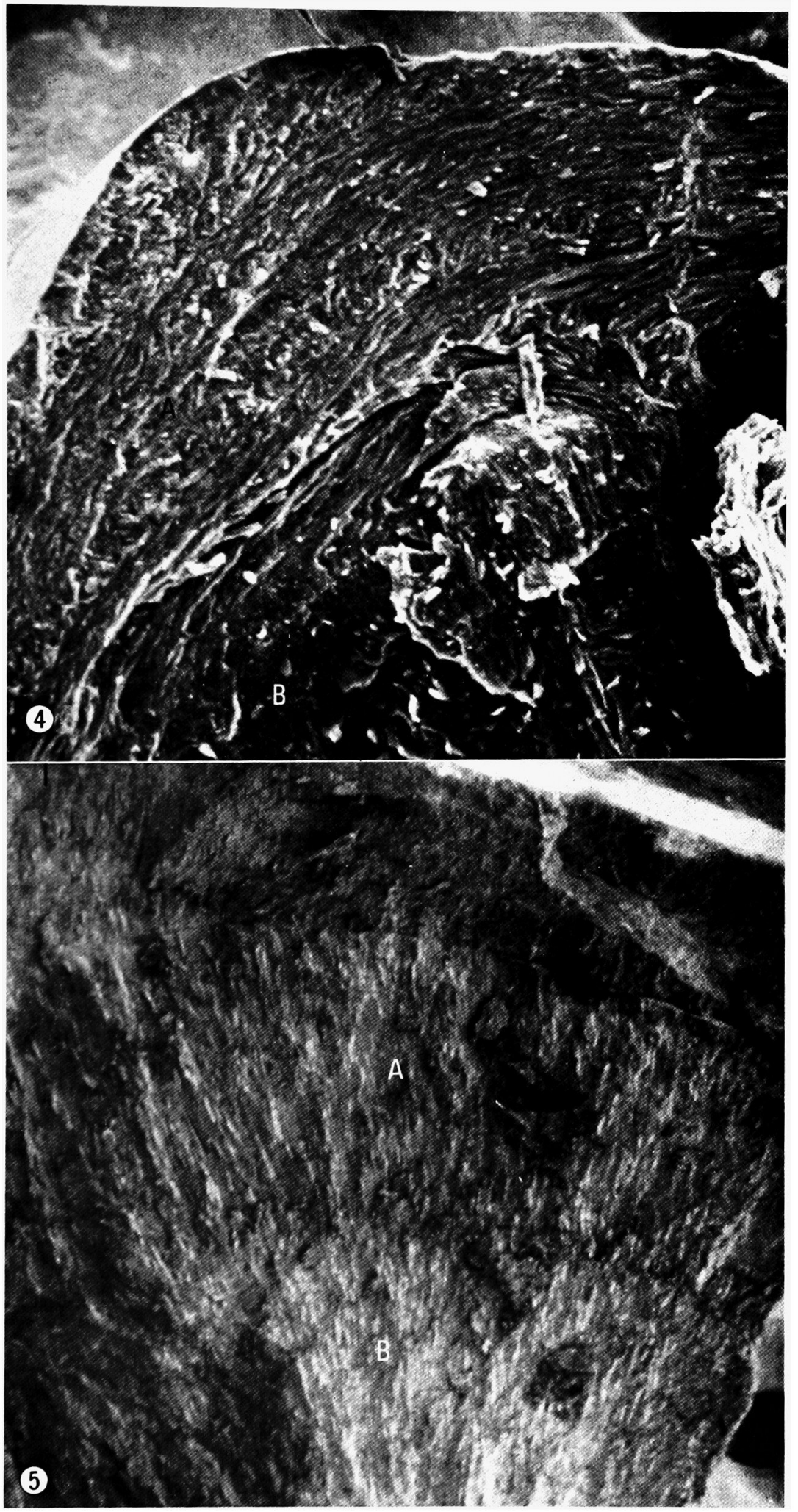


Fig. 6. A general view at lower magnification of the external surface of the stone shown. Fig. 1. It appears a group of gently sloping domes. At the boundary between two domes, plate-like crystals are arranged perpendicular to the surface. $\times 200$.

Fig. 7. External surface of the stone in Fig. 12. Various sized crystals shaped in parallelogram-bricks of which the obtuse angle is about $120^{\circ}$ are seen over the surface. Note examples of a small crystal being generated from a large base one (arrows). $\times 1,100$. 


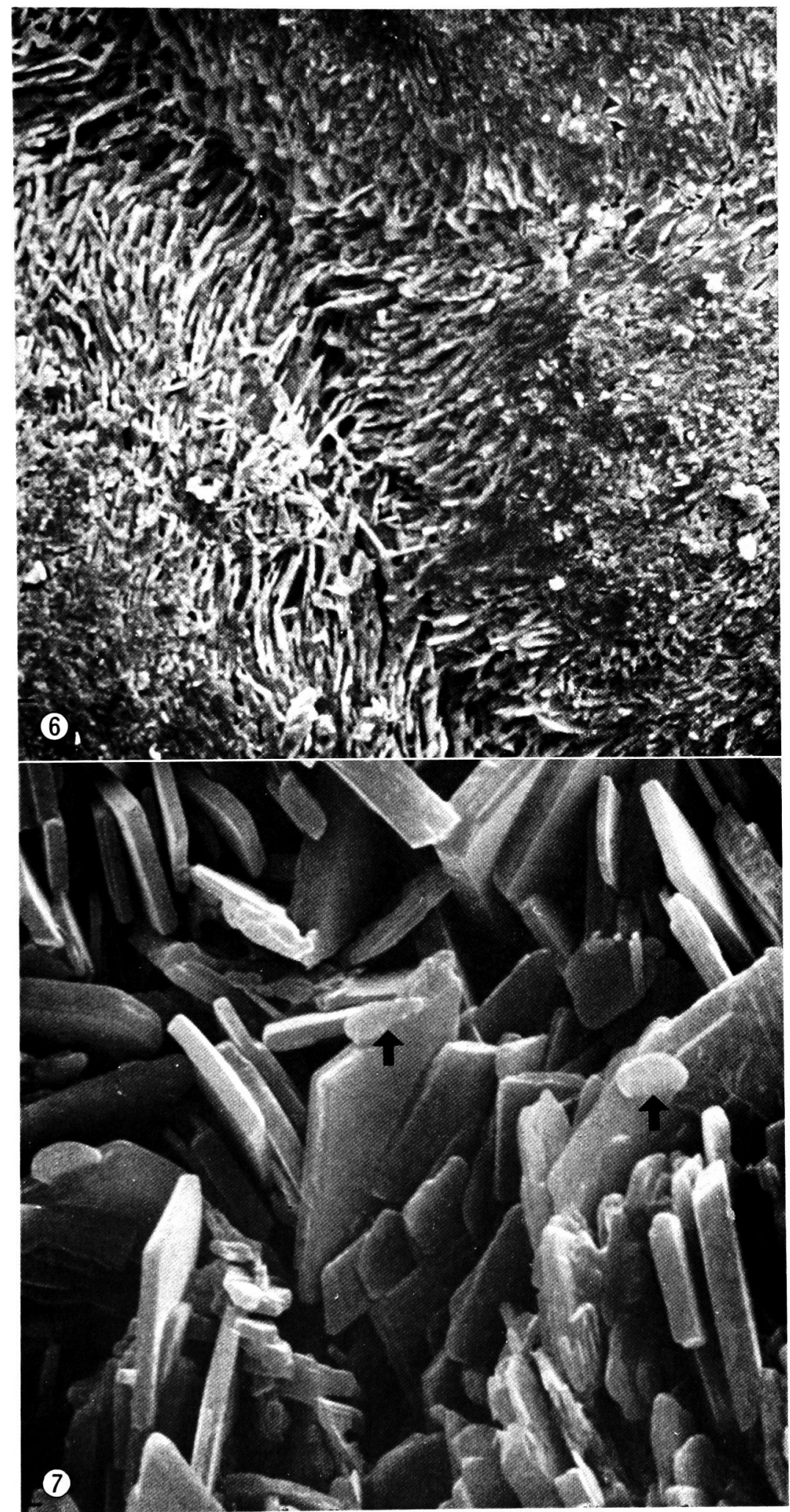


Figs. 8 to 11. External surface of a radiate cholesterol stone about $2 \mathrm{~cm}$ in diameter obtained from a 16-year-old female.

Fig. 8. Note the columnar projections arranged like cogs on a cogwheel, each projection consisting of multilayered cholesterol crystal sheets. Smaller crystals (B, C and D) generate from a large base one $(\mathrm{A}) . \times 120$.

Fig. 9. On the surface of a cholesterol crystal, lamellae are observed. $\times 1,200$. 


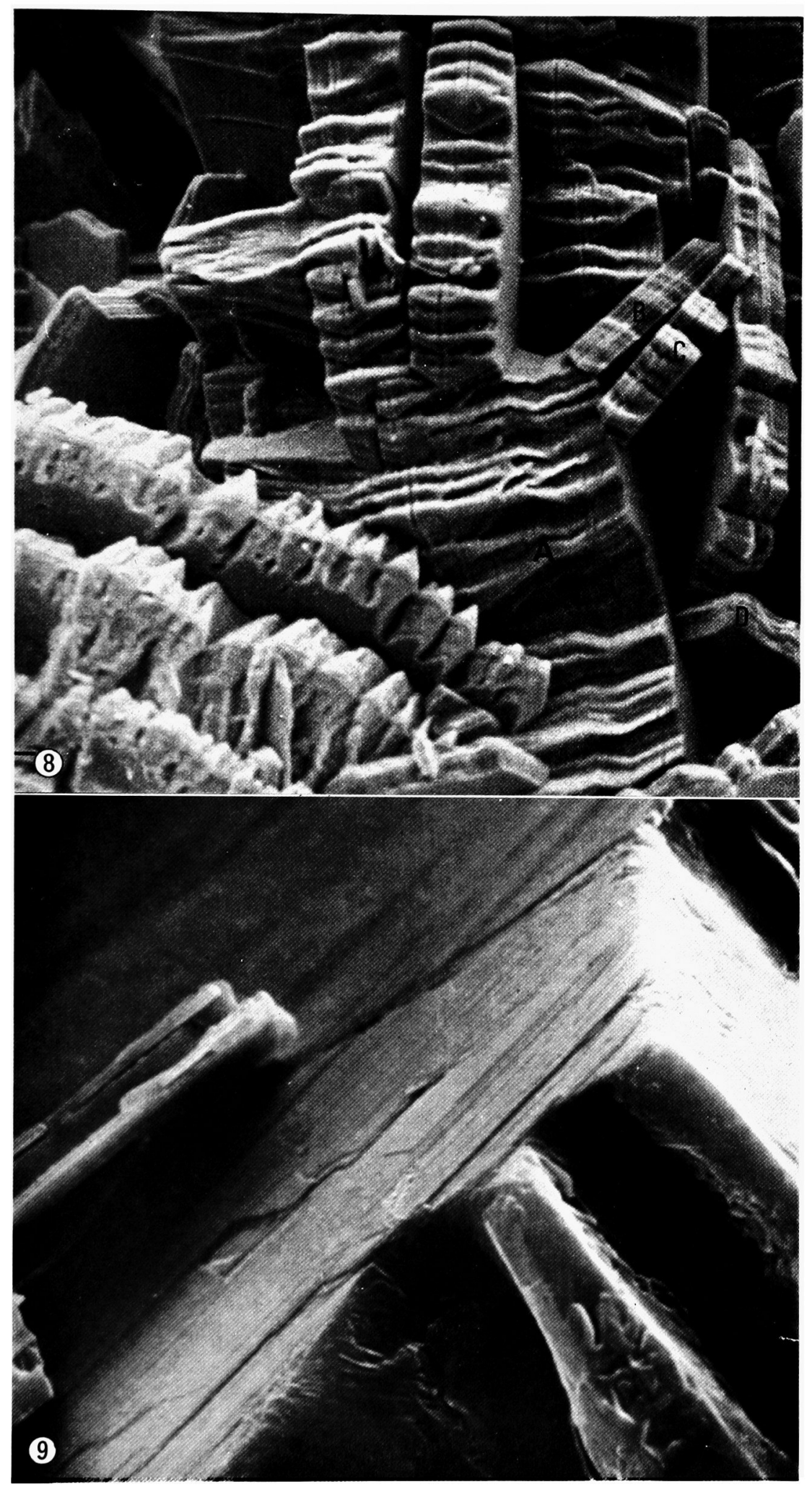


Fig. 10. Illustrating the phenomenon of "spiral growth". This regular circular spiral shape appears on the surface of a cholesterol crystal. $\times 13,700$.

Fig. 11. Higher magnification of a part of Fig. 10. The ledge starting from $\mathbf{A}$ goes on rotating and generating the spiral. The step height appears to be about $200 \mathrm{~A}$ in thickness. $\times 43,500$. 


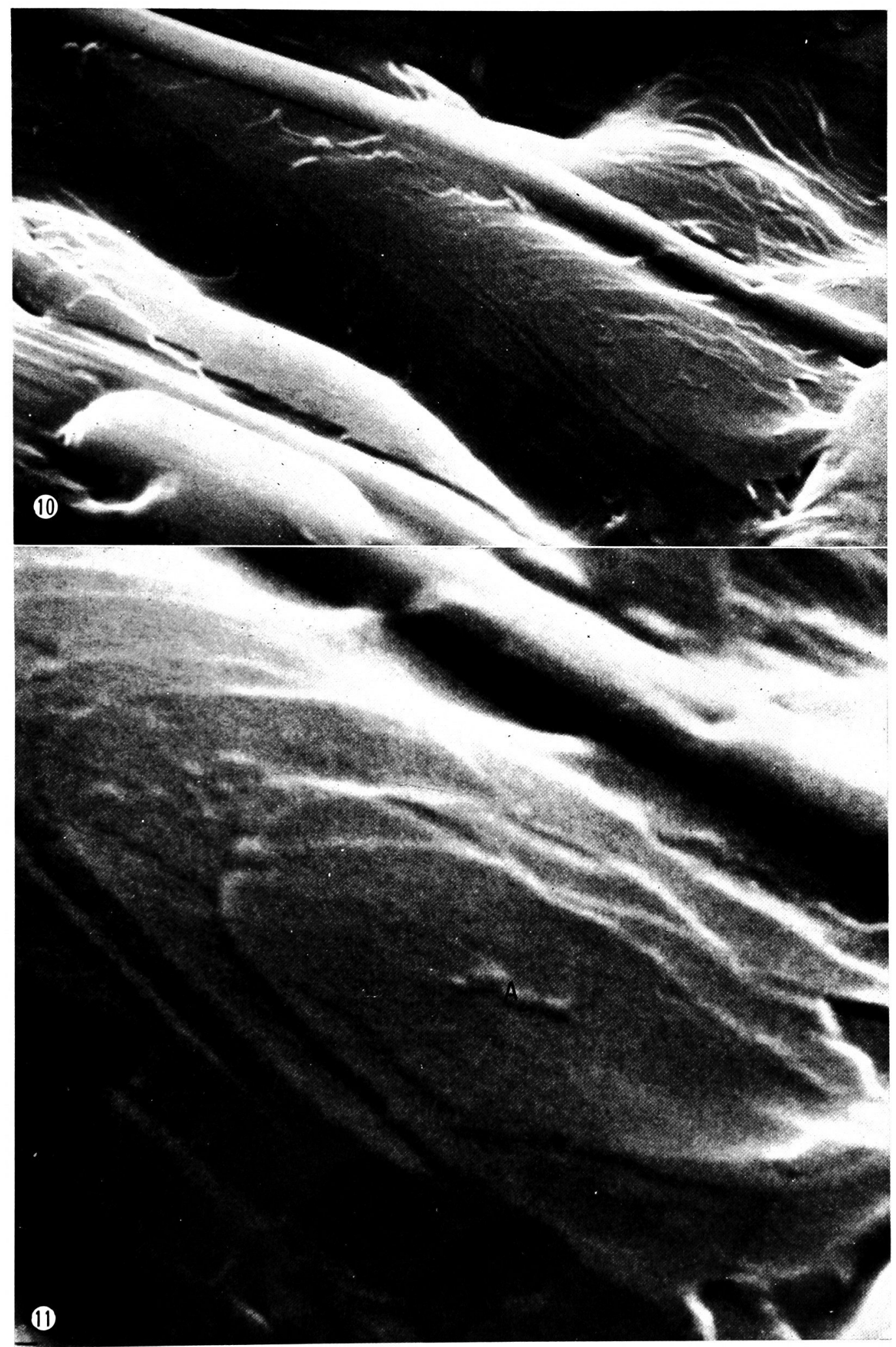


Fig. 12. This fracture plane of a multinuclear stone about $2.5 \mathrm{~cm}$ in diameter, obtained from the gallbladder of a 40 -year-old female. Note that the crystallization which spreads out from the two nuclei ( $\mathrm{Nl}$ and N2) stops proceedings over the common portion, where between $A$ and $B$, the two ledges meet and form a straight line: $\times 40$.

Fig. 13. Higher magnification of boundary area of Fig. 12. Note the edge of numerous lamellae which join together without mutual interference. $\times 670$. 


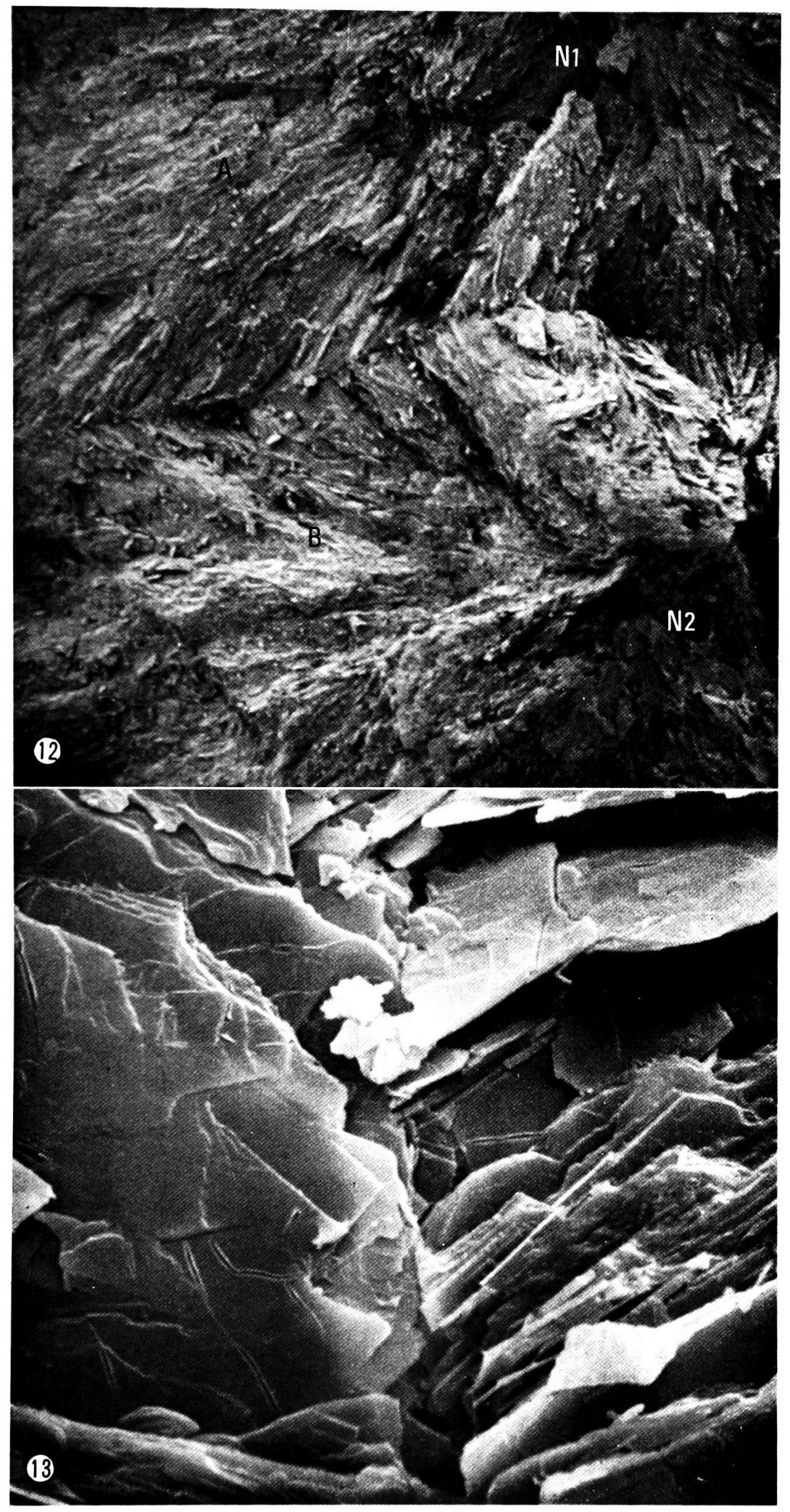




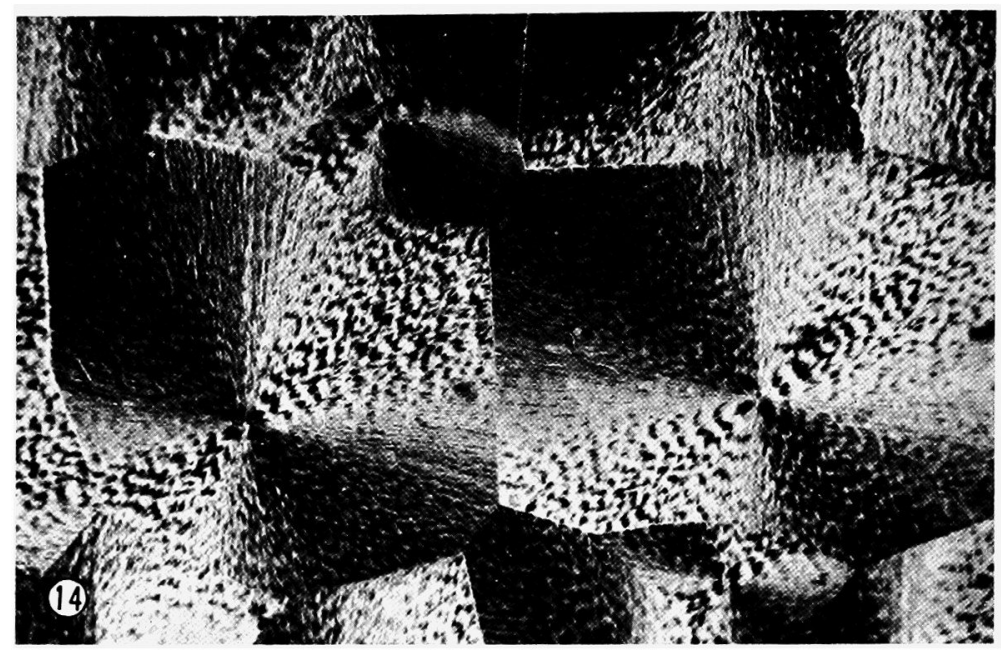

Fig. 14. Spherulite of high density polyethylene crystallized from a molten thin film. $\times 200$. 